\title{
A New Method for Cylindrical Multilayered Waveguides Analysis
}

\author{
Eduard E. Malov, Yuriy E. Mitelman and Sergey N. Shabunin \\ Institute of Radioelectronics and Information Technologies, \\ Ural Federal University, Ekaterinburg, Russian Federation
}

\begin{abstract}
Cylindrical multilayered waveguides are often used as parts of various microwave and optical devices. Layered structure of waveguide filling allows more flexibility of tuning and optimization of the device parameters. In the waveguide with inhomogeneous cross-section, one can obtain order of cut-off frequencies of eigen modes which is different from the normal one. The study describes a new technique of obtaining the dispersion characteristics of such waveguides based on tensor Green's functions method. The proposed method is effective in constructing the dispersion equations as it uses microwave circuits theory description and recurrent expressions instead of direct solution of the system of boundary equations.
\end{abstract}

Key words: Waveguides, propagation constant, transmission lines, Green's function methods, analysis

\section{INTRODUCTION}

Waveguides are the basic structures for microwave resonators, filters, rotary joints, etc. Waveguides with inhomogeneous filling can be used to obtain, e.g., low dispersion and attenuation in transmission line, high-Q resonators or can serve as parts of mode filters. The particular form of these filling is radially piecewise inhomogeneous cylindrical waveguides such as circular waveguides, coaxial lines, optical fibers and Goubau lines. One of the main characteristics of such structures is a propagation constant (wave number) which contains the information about phase constant and attenuation. The most popular for analysis of multilayered waveguides is the method in which boundary conditions between homogeneous layers are used to get unique solution of differential wave equation. These boundary equations form a system which can be solved for wave number as unknown. With the increasing number of layers in the waveguide the order of that system increases thus increasing its complexity and its solution becomes more complicated. Therefore, the time of computation rises dramatically either the accuracy of the solution decreases with the raise of the complexity of the structure. However, some papers describe waveguides with continuously inhomogeneous dielectric filling (Kiang, 1996) but only for axisymmetric modes.

\section{MATERIALS AND METHODS}

The proposed method uses Green's functions method for dispersion equations composing in cylindrical radially piecewise homogeneous waveguides. This method is described by Knyazev et al. (2011) and is successfully implemented by Abdullin and Mitelman (2014) and (Abdullin et al., 2014) for radiation and propagation problems in rectangular slotted waveguide.

The idea is based on the Fourier integral-series spatial decomposition of the electric and magnetic field's components in the cylindrical structures:

$$
\begin{aligned}
& \mathrm{E}_{z}=\frac{1}{2 \pi} \sum_{\mathrm{m}=-\infty}^{\infty} \mathrm{e}^{-\mathrm{j} m \varphi} \int_{-\infty}^{\infty} \mathrm{E}_{z m h}(r) \mathrm{e}^{-\mathrm{jhz} d h} \\
& \mathrm{H}_{z}=\frac{1}{2 \pi} \sum_{\mathrm{m}=-\infty}^{\infty} \mathrm{e}^{-\mathrm{j} m \varphi} \int_{-\infty}^{\infty} \mathrm{H}_{z m h}(r) \mathrm{e}^{-\mathrm{jh} z} \mathrm{dh}
\end{aligned}
$$

Where:

$\mathrm{m}=$ The discrete azimuthal index of mode

$\mathrm{h}=$ The continuous wavenumber

Describing electromagnetic field as the sum of electrical and magnetic waves propagating along $\mathrm{z}$ direction we can convert wave equations to the system of two equations. For example for TM (E) waves:

$$
\begin{aligned}
& -\frac{\mathrm{d}}{\mathrm{dr}}\left(\mathrm{E}_{z \mathrm{mh}}^{\mathrm{e}}\right)=-\mathrm{j} \frac{\gamma^{2} \mathrm{Z}_{0}}{\varepsilon \mathrm{rk}_{0}^{2}}\left(\mathrm{k}_{0} \mathrm{rH}_{\varphi \mathrm{mh}}^{\mathrm{e}}\right)-\frac{\mathrm{hZ_{0 }}}{\varepsilon \mathrm{k}_{0}} \mathrm{~J}_{\mathrm{rmh}}^{\mathrm{E} e x}-\mathrm{J}_{\phi \mathrm{mh}}^{\mathrm{M} e x} \\
& \frac{\mathrm{d}}{\mathrm{dr}}\left(\mathrm{k}_{0} \mathrm{rH}_{\varphi \mathrm{mh}}^{\mathrm{e}}\right)=\mathrm{j} \frac{\mathrm{\varepsilon k} \mathrm{k}_{0}^{2}}{\mathrm{Z}_{0} \gamma^{2}} \mathrm{r}\left[\mathrm{k}^{2}-\mathrm{h}^{2}-\left(\frac{\mathrm{m}}{\mathrm{r}}\right)^{2}\right] \mathrm{E}_{z \mathrm{mh}}^{\mathrm{e}}+ \\
& \mathrm{k}_{0} \mathrm{rJ}_{z \mathrm{mh}}^{\mathrm{E} e x}-\frac{\mathrm{mhk}_{0}}{\gamma^{2}} \mathrm{~J}_{\varphi \mathrm{mh}}^{\mathrm{E} e x}+\frac{\varepsilon \mathrm{k}_{0}}{\gamma^{2} Z_{0}} \mathrm{~mJ}_{\mathrm{rmh}}^{\mathrm{Mex}}
\end{aligned}
$$

Corresponding Author: Yuriy E. Mitelman, Institute of Radioelectronics and Information Technologies, Ural Federal University, Ekaterinburg, Russian Federation 


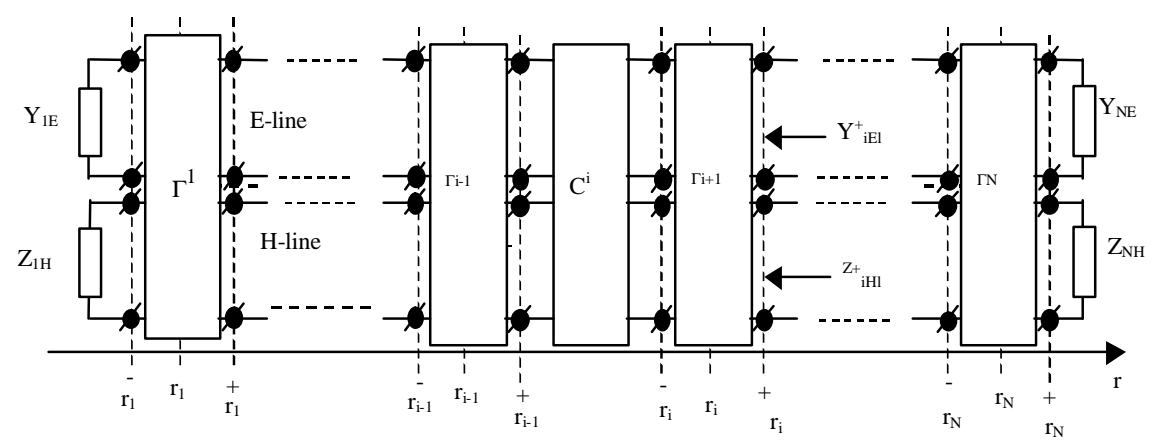

Fig. 1: Equivalent circuit of the radial lines

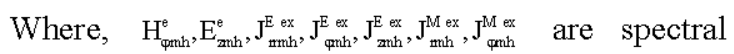
components of the electric and magnetic fields, electric and magnetic external currents respectively, Y- transverse wave number. With interchange, $\mathrm{V}_{\mathrm{E}}=\dot{\mathrm{E}}_{z \mathrm{mh}}^{e}, \mathrm{I}_{\mathrm{E}}=-\mathrm{k}_{0} \mathrm{r} \dot{\mathrm{H}}_{\text {qmh }}^{e}$, we can regress Eq. 3 to system of telegraph equations:

$$
\begin{aligned}
& -\frac{d}{d r} V_{E}=j Z_{E} \chi I_{E}+v_{e x}^{E} \\
& -\frac{d}{d r} I_{E}=j Y_{E} \chi V_{E}+i_{\text {ex }}^{E}
\end{aligned}
$$

Where, $V_{E}, I_{E}, Z_{E}, Y_{E}$ are voltage, current, impedance and admittance of the equivalent radial $E$-line, $v_{e x}$ and $i_{e x}$ are equivalent voltage and current of sources $\chi=\sqrt{\mathrm{k}^{2}+\mathrm{h}^{2}-(\mathrm{m} / \mathrm{r})^{2}}$ is the propagation constant in the equivalent radial transmission line. The same equations we can obtain for magnetic $\mathrm{H}$-lines. Thus using the methods of circuits theory we can replace inhomogeneous structure with equivalent circuit consisting of two equivalent radial lines (Fig. 1).

Boundaries and layers in this circuit can be characterized by their transmission matrices $\Gamma$ and $\mathrm{C}$ respectively which connect the voltages and currents on their ports. In the same way, we can describe the ends of these lines using impedances $Z_{1 \mathrm{H}}, Z_{\mathrm{NH}}$ and admittances $\mathrm{Y}_{1 \mathrm{E}}, \mathrm{Y}_{\mathrm{NE}}$ corresponding to the internal and external layers of the structure.

The described model can be used for example for field calculations in every layer for eigen modes of the multilayered circular waveguide. In this study, we apply this model to a circular metal layered waveguide and calculate its dispersion characteristics.

\section{RESULTS AND DISCUSSION}

To obtain the propagation constant of the waveguide we can use cross resonance conditions at the section $p$ of radial line formulated as equations with directional modal impedances and admittances (Walter, 1990):

$$
\begin{aligned}
& Z_{\mathrm{pHI}}(\mathrm{m}, \mathrm{h})+\mathrm{Z}_{\mathrm{pHr}}(\mathrm{m}, \mathrm{h})=0 \\
& \mathrm{Y}_{\mathrm{pEl}}(\mathrm{m}, \mathrm{h})+\mathrm{Y}_{\mathrm{pEr}}(\mathrm{m}, \mathrm{h})=0
\end{aligned}
$$

The first equation solution is suitable for TM (E) modes and the second is for TE $(\mathrm{H})$ modes. One should solve these equations for $\mathrm{h}$ as unknown on a particular frequency.

Described method allows creating an algorithm for dispersion analysis of cylindrical transmission lines with theoretically unlimited number of layers. Created program is able to calculate cut-off frequencies of eigen waves and plots of the propagation constant versus frequency in the cylindrical metal waveguide with 12 layers. Adding a new layer will lead to a non-significant increasing of computational time because in that case one should add only two more two-port devices in the equivalent circuit. To evaluate the operating frequency range of the waveguide, usually the concept of the Fractional Band Width (FBW) coefficient (the ratio between the critical frequencies of the first higher mode and the fundamental mode) is used:

$$
\mathrm{T}=\frac{\mathrm{f}_{\mathrm{c}}^{\mathrm{h}}}{\mathrm{f}_{\mathrm{c}}^{\mathrm{f}}}
$$

The following types of cylindrical waveguides: empty waveguide, uniformly filled waveguide, waveguide with dielectric rod with dielectric cylinder with an air gap in the filling, with parabolic dielectric profile were analyzed.

$\mathrm{TE}_{11}\left(\mathrm{H}_{11}\right)$ wave is the fundamental wave in a cylindrical uniformly filled waveguides (Fig. 2 and 3 ). FBW in empty and filled by dielectric waveguides is $\mathrm{t}=1.306$. When using uniform dielectric filling, wavelengths of all modes decrease by $\sqrt{\varepsilon}$. It means that usage of uniformly filled dielectric is helpful for miniaturization of waveguides. In addition the phase velocity and impedance decrease. To avoid high attenuation due to the dielectric losses one needs to use an expensive high quality materials. The y-axis of all 


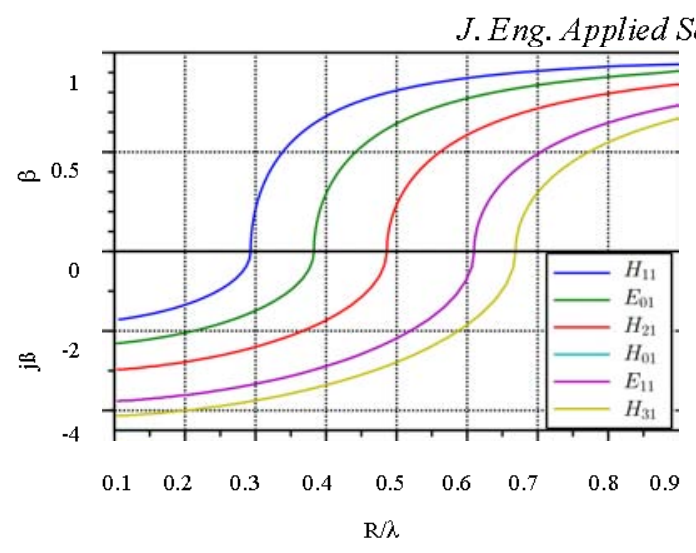

Fig. 2: Propagation constant of the eigen modes in the empty waveguide

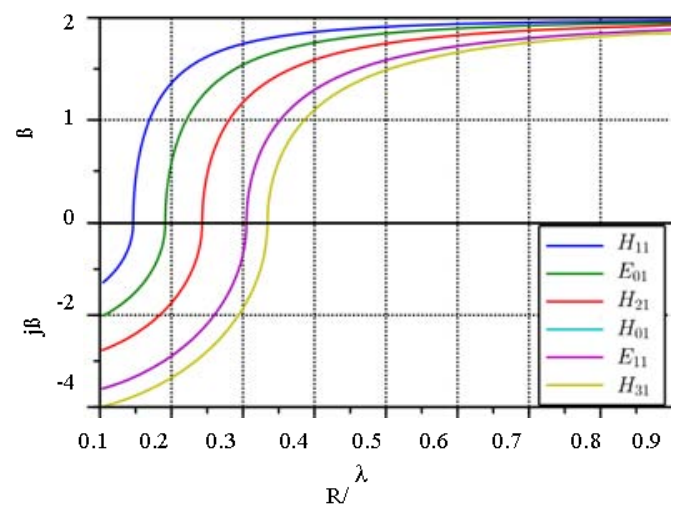

Fig. 3: Propagation constant of the eigen modes in the uniformly filled waveguide

frequency dependencies is normalized wave number. $\mathrm{E}_{01}$ mode is often being used in devices with rotating joint waveguides. Electromagnetic field must be uniform along the azimuthal coordinate. $\mathrm{E}_{01}$ wave is the first higher order mode in empty waveguide. To use this wave as operating one it is needed to suppress the fundamental wave with special mode filter. With using filters one can achieve FBW coefficient for this mode $t=1.27$.

It is possible to create propagation conditions for $\mathrm{E}_{01}$ wave to make it the fundamental wave. To do this, a non-uniform dielectric filling can be used. Figure 4 and 5 show dependences for a waveguide with dielectric rod in the center. FBW coefficient becomes $\mathrm{t}=1.04$ (Fig. 2) without usage of special filters to suppress the $\mathrm{HE}_{11}$ wave. However, in the case we can suppress $\mathrm{HE}_{11}$ wave, coefficient increases to $\mathrm{t}=1.89$ and this value is quiet big for cylindrical waveguide. Using a larger value of permittivity (Fig. 5) increased FBW coefficient of the $\mathrm{E}_{01}$ mode as fundamental wave to $\mathrm{t}=1.4$ without filters and to $\mathrm{t}=1.97$ with suppressing of the hybrid mode $\mathrm{HE}_{11}$.

The examples show that filling parameters could be chosen the way in which one can change the properties of the transmission line including fundamental mode.

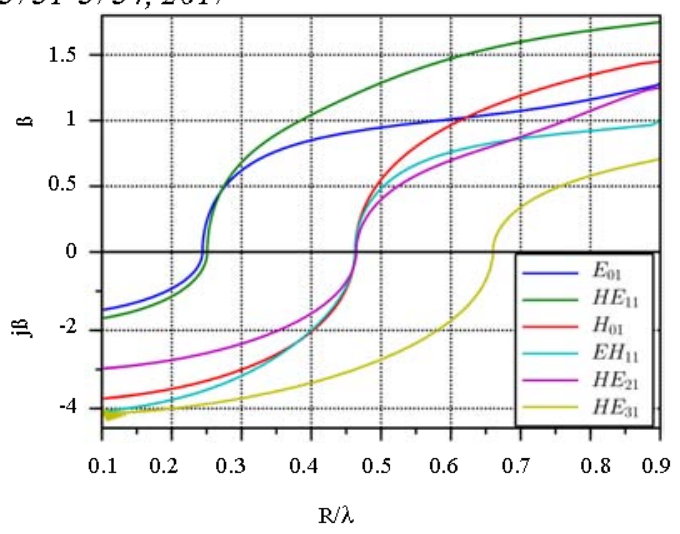

Fig. 4: Propagation constant of the eigen modes in the waveguide with dielectric $\operatorname{rod}(\varepsilon=4)$

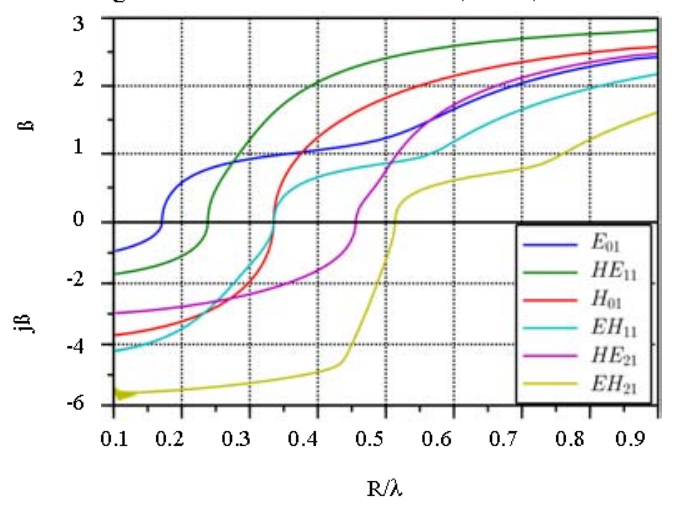

Fig. 5: Propagation constant of the eigen modes in the waveguide with dielectric $\operatorname{rod}(\varepsilon=9)$

This effect was demonstrated in the past, i.e., in (Tsandoulas and Ince, 1971). In addition, dielectric rod allowed decreasing the dispersion for $\mathrm{E}_{01}$ and all $\mathrm{EH}_{\mathrm{m} 1}$. Dispersion decreasing of fundamental wave mode allows transmitting signals with low distortions.

The use of $\mathrm{H}_{01}$ mode in circular waveguides is very attractive in terms of low attenuation. In the uniformly filled metal cylindrical waveguide $\mathrm{H}_{0 \mathrm{n}}$ and $\mathrm{E}_{\mathrm{n} 1}$ waves have the same cut-off frequencies and their frequency dependences of wave number are identical. This point makes it more difficult to use $\mathrm{H}_{01}$ mode because it is scattered by randomly located discontinuities in the waveguide and converted into a spectrum of waves with small amplitudes including $\mathrm{E}_{01}$ wave. Since, the speed of propagation of $\mathrm{H}_{01}$ and $\mathrm{E}_{11}$ modes are very close the amplitude of the $\mathrm{E}_{11}$ wave tends to accumulate when other amplitudes still small, so $\mathrm{H}_{01}$ wave constantly converts into $\mathrm{E}_{11}$ wave.

In inhomogeneous waveguides cut-off frequencies of $\mathrm{H}_{01}$ and $\mathrm{EH}_{11}$ modes $\left(\mathrm{E}_{11}\right.$ becomes $\left.\mathrm{EH}_{11}\right)$ are still equal but propagation constants become different. It creates conditions for more stable propagation of $\mathrm{H}_{01}$ mode. 


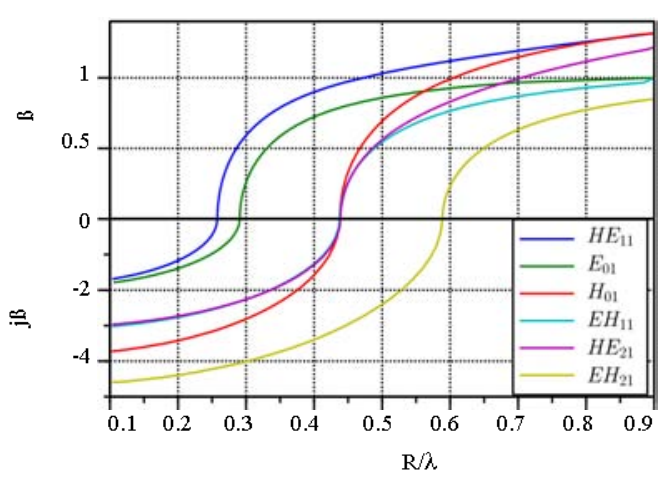

Fig. 6: Propagation constant of the modes in the waveguide with dielectric cylinder

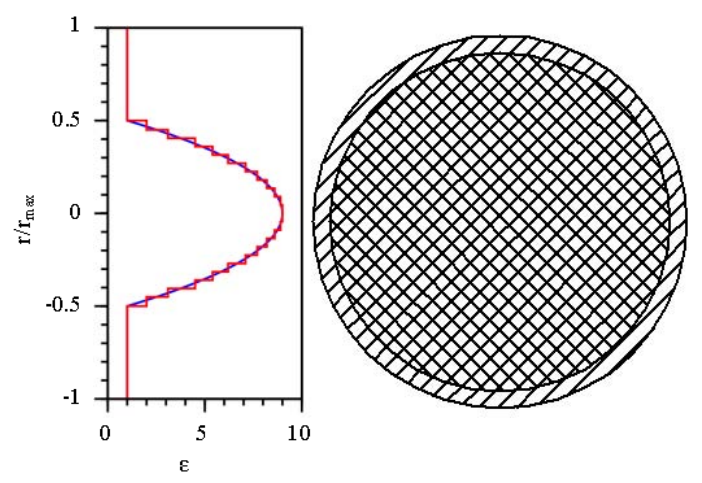

Fig. 7: Parabolic dielectric profile of the complex filled waveguide

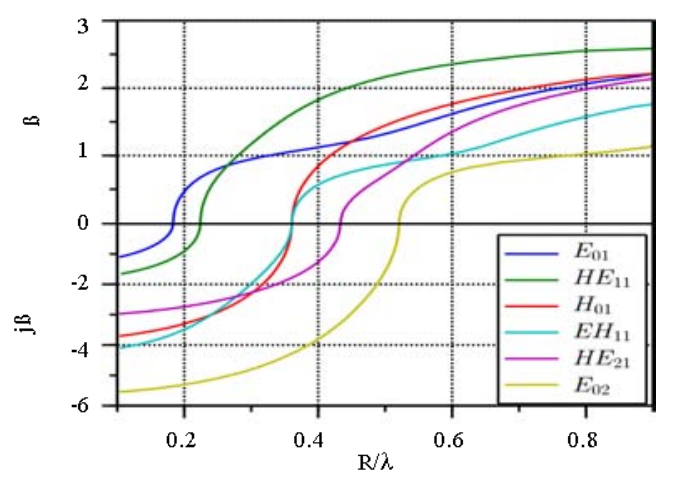

Fig. 8: Propagation constant of the modes in waveguide with parabolic dielectric profile

Splitting of $\mathrm{H}_{01}$ and $\mathrm{EH}_{11}$ is possible in all nonuniformly filled structures. The best result in splitting can be obtained using dielectric cylinder (Fig. 6).

Created software allows calculating characteristics of the structures with up to 12 layers. Non-uniform dielectric profile (Fig. 7) can be approximated by a stepped function, so the described method can be useful in stratified filling researches. The results of simulation are shown in Fig. 8.

\section{CONCLUSION}

In the study, the novel effective method for solving the problems of dispersion calculation of the circular metal waveguides with inhomogeneous filling is described. It is shown that calculation not only the phase constant but also an attenuation of cut-off modes and overmoded waveguides is possible. Continuous dielectric profiles can be approximated as accurately stratified media. In that case, the efficiency of the method is provided by its matrix nature and using of the recurrent equations of the circuit theory.

\section{ACKNOWLEDGEMENT}

The research was executed by the grant of the Russian Science Foundation (Project No. 14-19-01396).

\section{REFERENCES}

Abdullin, R.R. and Y.E. Mitelman, 2014. Using of partially filled rectangular waveguide for leaky-wave antenna design. Proceeding of the 2014 24th International Conference on Crimean Microwave and Telecommunication Technology (CriMiCo), September 7-13, 2014, IEEE, Yekaterinburg, Russia, ISBN:978-966-335-412-5, pp: 543-544.

Abdullin, R.R., Y.E. Mitelman and S.N. Shabunin, 2014. Velocity factor of leaky-wave antenna based on partially filled rectangular waveguide. Proceeding of the 2014 12th International Conference on Actual Problems of Electronics Instrument Engineering (APEIE), October 2-4, 2014, IEEE, Yekaterinburg, Russia, ISBN:978-1-4799-6019-4, pp: 406-409.

Kiang, J.F., 1996. Axisymmetric modes in an inhomogeneous circular waveguide. Proceeding of the International Symposium on AP-S: Digest Antennas and Propagation Society, July 21-26, 1996, IEEE, Taichung, Taiwan, ISBN:0-7803-3216-4, pp: 976-979.

Knyazev, S., L. Lesnaya and S. Shabunin, 2011. Greens functions of multilayered cylindrical structures and their application for radiation, propagation and scattering problems solving. Proceeding of the 2011 SBMO/IEEE International Conference on MTT-S Microwave and Optoelectronics (IMOC), October 29-November 1, 2011, IEEE, Yekaterinburg, Russia, ISBN:978-1-4577-1662-1, pp: 748-752.

Tsandoulas, G.N. and W.J. Ince, 1971. Modal inversion in circular waveguides Part I: Theory and phenomenology. IEEE. Trans. Microwave Theor. Tech., 19: 386-392.

Walter, C.H., 1990. Traveling Wave Antennas. McGraw-Hill Education, Los Altos, California. 